\title{
Acute Retinal Necrosis Secondary to Varicella Zoster Virus in an Immunosuppressed Post-Kidney Transplant Patient
}

\author{
Elizabeth Chiang, MD, PhD and Dmitry Pyatetsky, MD
}

A

woman, aged 42 years, presented to the ophthalmologist complaining of right eye redness, tearing, photosensitivity, and blurred vision of a 1-month duration. Her past medical history was significant for a kidney transplant for which she was immunosuppressed with mycophenolate mofetil $750 \mathrm{mg}$ twice a day and prednisone $10 \mathrm{mg}$ daily.

Ophthalmologic examination of the right eye revealed visual acuity of 20/60, intraocular pressure of $16 \mathrm{mmHg}$, scleral injection, and a significant inflammatory reaction in the anterior chamber. Funduscopy revealed vitreous haze, optic nerve

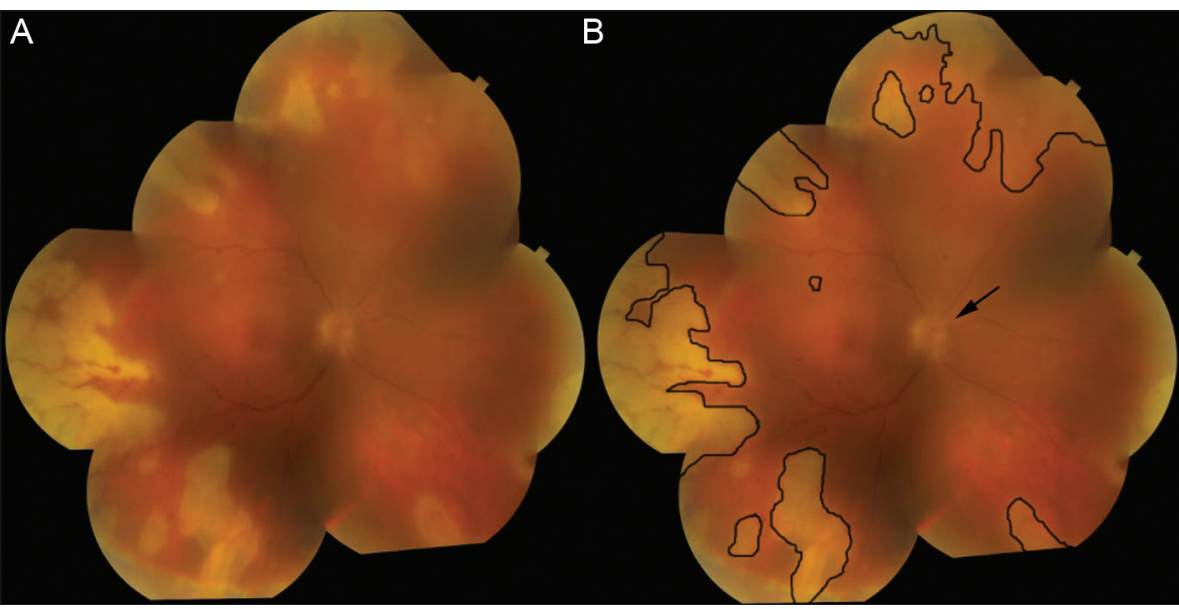

Figure 1. (A) Examination of the fundus on day of presentation shows peripheral whitening and vasculitis as evidenced by optic nerve edema, vitreous haze, and retinal arteriolar narrowing. The decrease in clarity of the photograph is secondary to the vitreous haze, indicating a severe intraocular immune response in the form of vitritis. (B) The peripheral whitening is indicated by the areas outlined in black. The blurred margins of the optic nerve, indicated by the black arrow, suggest optic nerve edema. edema, arteriolar sheathing and attenuation, and peripheral $360^{\circ}$ of retinal whitening - a clinical picture consistent with retinitis (figure 1). The left eye examination was entirely normal.

The differential diagnosis for retinitis in an immunocompromised host includes acute retinal necrosis (ARN), progressive outer retinal necrosis, cytomegalovirus (CMV) retinitis, and toxoplasmosis. Less common causes of acute retinitis include syphilis, ${ }^{1}$ Beçhet's disease, and lymphoma.

Aqueous fluid obtained from the anterior chamber was sent for polymerase chain reaction (PCR) viral DNA analysis, and the patient was treated with $2 \mathrm{mg}$ of ganciclovir injected intravitreally on the day of
Corresponding Author:

Dmitry Pyatetsky, MD

Department of Ophthalmology

Northwestern University

645 North Michigan Ave, Suite 440

Chicago, IL 606II

Tel: (3।2) 908-8I52

Fax: (3।2) 503-8I52

Email: dpyatetsky@hotmail.com
Keywords: Acute retinal necrosis syndrome; Immunocompromised host; Immunosuppression; Kidney transplantation; Uveitis; Varicella zoster virus

Received: April 23, 2012

Revised: July 10, 2012

Accepted: July 18, 2012

doi: $10.3121 / \mathrm{cmr} .2012 .109$

The Aperture, like the opening in the lens of a microscope that allows light to pass through, is a forum for art, humor, and images that provides a portal for new or different views of medicine and research. 


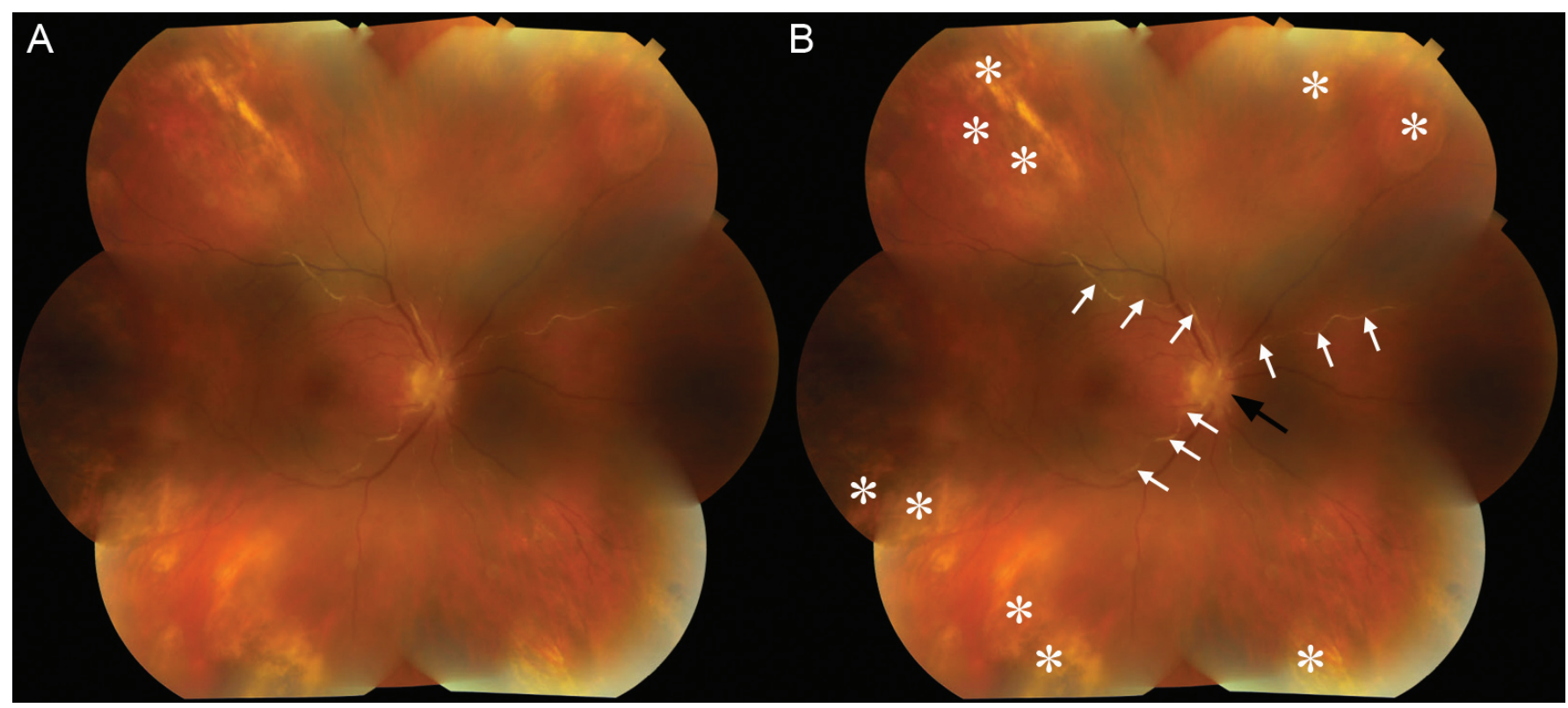

Figure 2. (A) After treatment with anti-viral medication, there is regression of retinal whitening and atrophic retinal tissue. (B) Areas of regression are indicated by white asterisks. The arterioles that previously were narrowed, indicated by the white arrows, now appear sclerotic. The optic nerve, indicated by the black arrow, continues to be edematous with blurred margins.

presentation. A 2-week course of renal-dose adjusted intravenous (IV) acyclovir was started with subsequent regression of the retinal whitening (figure 2). Varicella zoster virus (VZV) infection with a viral load of $6.5 \times 10^{7} \mathrm{IU}$ was later confirmed with PCR of the aqueous fluid. The vision initially improved to $20 / 40$ in the right eye. She was transitioned from IV acyclovir to oral valacyclovir.

Seven weeks later, the patient developed a rhegmatogenous retinal detachment that was diagnosed after she complained of an abrupt decrease in vision. Despite successful surgical reattachment of the retina with pars plana vitrectomy, scleral buckle, and silicon oil, the patient's vision remained at light perception level only.

Necrotizing herpetic retinopathies are caused by VZV, herpes simplex virus 1 and 2, CMV, and rarely, Epstein Barr virus. A spectrum of clinical presentations may occur depending on the virus and the host's immunity. ${ }^{2}$ Immunosuppressed patients are at risk for development of necrotizing retinopathies typically associated with immunocompromised states, such as CMV retinitis and progressive outer retinal necrosis. They may also develop syndromes that are typically seen in immunocompetent hosts, such as ARN.

Acute unilateral decrease in vision, photophobia, and eye pain are the usual presentations of $A R N .{ }^{3}$ The key feature seen on examination is white-yellow, multifocal, peripheral patches that later coalesce into diffuse areas of full-thickness, peripheral retinal necrosis. ${ }^{4}$ Other signs of ocular inflammation including vitritis, vasculitis, optic disc edema, keratic precipitates, and posterior synechiae may also be seen. Untreated ARN results in blindness from retinal scarring, retinal detachment, or optic atrophy; therefore, ARN is an ophthalmic emergency. One-third of patients develop the disease in the fellow eye within one month of presentation, if left untreated. ${ }^{5}$ The diagnosis of ARN is made on the basis of clinical appearance, and antiviral treatment should be initiated promptly. Timely referral to a uveitis or retina specialist experienced in the care of such patients and collaboration with an infectious disease specialist are essential.

\section{References}

1. Gupta G, Pyatetsky D. Posterior uveitis secondary to syphilis. Clin Med Res 2009;7:106.

2. Wensing B, de Groot-Mijines JD, Rothova A. Necrotizing and nonnecrotizing variants of herpetic uveitis with posterior segment involvement. Arch Ophthamol 2011;129:403-408.

3. Muthiah MN, Michaelides M, Child CS, Mitchell SM. Acute retinal necrosis: a national population-based study to assess the incidence, methods of diagnosis, treatment strategies and outcomes in the UK. Br J Ophthalmol 2007;91:452-455.

4. Holland GN. Standard diagnostic criteria for the acute retinal necrosis syndrome. Executive Committee of the American Uveitis Society. Am J Ophthamol 1994;117:663-667.

5. Palay DA, Sternberg P Jr, Davis J, Lewis H, Holland GN, Mieler WF, Jabs DA, Drews C. Decrease in the risk of bilateral acute retinal necrosis by acyclovir therapy. Am J Ophthalmol 1991;112:250-255.

\section{Author Affiliations}

Elizabeth Chiang, MD, PhD*; Dmitry Pyatetsky, MD*

*Department of Ophthalmology, Feinberg School of Medicine, Northwestern University, Chicago, Illinois USA 